\title{
Medicolegal Issues in Obstetric and Gynecologic Ultrasound
}

Frank A Chervenak, Judith L Chervenak

\begin{abstract}
Professional liability risks exist in the practice of ultrasound. In this article, basic aspects of medical negligence are reviewed. The guidelines published by ACOG and AIUM are presented and discussed relating to the importance of instrumentation and safety, documentation, and indications for first, second and third trimester ultrasound examinations as well at the content of these examinations. Litigation related to ultrasound is presented and the controversial nonmedical use of ultrasonography is discussed.
\end{abstract}

Keywords: Professional liability, Malpractice, Medical negligence, Guidelines, Litigation.

How to cite this article: Chervenak FA, Chervenak JL. Medicolegal Issues in Obstetric and Gynecologic Ultrasound. Donald School J Ultrasound Obstet Gynecol 2012;6(1):9-13.

Source of support: Nil

Conflict of interest: None declared

\section{INTRODUCTION}

Ultrasound has revolutionized the practice of obstetrics and gynecology in first generation more than any other innovation. However, practitioners should be aware of the medicolegal risks.

Obstetric ultrasound plays a vital and increasingly frequent role in legal actions, either as the focus in a case alleging wrongful birth in which an anomaly was not diagnosed and the mother was deprived of a chance to terminate her pregnancy; or as a significant or contributing factor in a case alleging negligent obstetric care with resulting damage to the infant-plaintiff or mother.

This chapter will focus upon the general aspects of a medical negligence case as they relate to the performance of the obstetric ultrasound examination, summarize the recommendations of both the American College of Obstetrics and Gynecology (ACOG) and the American Institute of Ultrasound in Medicine (AIUM), regarding the performance of these examinations, and outline potential areas of negligence and discuss ways to avoid them.

\section{MEDICAL NEGLIGENCE}

In order to establish negligence, the plaintiff must show that there was:

- A duty recognized by the law

- A breach of that duty, in that there was a failure on the part of the physician to meet what was considered to be the standard of care at the time the treatment was rendered

- A causal relationship between the treatment and the resulting injury, and

- Actual loss or damage to the plaintiff. ${ }^{1}$

Obstetric ultrasound cases may include allegations of a failure on the part of the maternal-fetal medicine specialist performing the ultrasound to fully advise the obstetric patient regarding the medical aspects of her case, given his specialized training in the field of high- risk obstetrics. The maternal-fetal medicine specialist has a duty to the patient and should clearly define the extent of his role in the patient's care, whether he or she is co-managing a patient, rendering consultative services or only performing antenatal diagnoses.

Generally, damages are easily established, granting either or both the departure from the accepted standards of care and the causal connection between that breach and the damages as the major focus of the litigation. Standard of care is most commonly established by the testimony of an expert witness whose knowledge, training or experience qualifies him to testify as to the standard of care. ${ }^{2}$ These experts are limited by the state of medical knowledge and standards of practice at the time of the alleged negligence. ${ }^{2}$ While these standards were previously limited to local legal requirements, they have expanded to those practiced nationally, given the recent advances in communication and dissemination of medical information.

Although guidelines promulgated by various organizations do not establish the standard of care introduced at trial, the obstetric ultrasound practitioner should be aware of the recommendations of ACOG and the AIUM. These organizations have published recommendations regarding guidelines, instrumentation and safety, documentation, indications, examination content and quality control. They periodically issue clinical recommendations. These guidelines are designed to inform the practitioner, so that he or she is aware of currently suggested practices in this ever-evolving discipline.

\section{GUIDELINES}

ACOG's recent publications include a Practice Bulletin entitled Ultrasonography in Pregnancy issued in December of $2004^{3}$ and a Committee Opinion on Guidelines for 
Diagnostic Imaging During Pregnancy in September of 2004. ${ }^{4}$ In 2003, the AIUM published a Practice Guideline for the Performance of an Antepartum Obstetric Ultrasound Examination in conjunction with ACOG and the American College of Radiology (ACR). ${ }^{5}$ The AIUM guidelines were originally published in 1985 and are now in their fourth revision.

\section{INSTRUMENTATION AND SAFETY}

While acknowledging that manufacturers offer machines with 3D capability, the practice bulletin indicates that proof of a clear advantage over 2D imaging has not yet been demonstrated. ${ }^{3}$ ACOG also recommends that practitioners should have a method of storing images and equipment should be serviced on a regular basis. ${ }^{3}$

The US Food and Drug Administration has arbitrarily limited energy exposure from ultrasonography to $94 \mathrm{~mW} \mathrm{~cm}{ }^{2}{ }^{4}$ In the 2004 Committee Opinion on Guidelines for Diagnostic Imaging During Pregnancy, ACOG noted that there had been no documented reports of adverse fetal effects from diagnostic ultrasound procedures, including duplex Doppler imaging. ${ }^{4}$ The AIUM concurs and emphasizes the 'as low as reasonably achievable' (ALARA) principle, which means that the lowest possible ultrasonic exposure setting should be used to gain the necessary information. ${ }^{5}$

\section{DOCUMENTATION}

The AIUM has published a standard for documentation of an ultrasound exam which can be obtained from the AIUM's website, www.aium.org. ${ }^{6}$ These guidelines recommend that a permanent record of both the images and the interpretation of the ultrasound be recorded in a retrievable format and should be kept in accordance with the relevant requirements of local legal and healthcare facilities. The AIUM suggests that the documentation include the patient's name and identifying numbers, such as a social security or medical record number, the date of ultrasound exam and image orientation on all recorded images. Additionally, the healthcare provider's name, type of ultrasound examination and identification of the sonographer/sonologist should be included on the accompanying report. ${ }^{6}$

A preliminary report of the findings may be provided and a final report should be included in the patient's medical record. Within the final report, limitations of the examination should be noted, biometric data, including variations from normal size, should be accompanied by measurements and a final report should be completed, and transmitted to the patient's healthcare provider. Depending on the circumstances, the results may need to be directly conveyed to the patient's referring health care provider and documentation of this communication is recommended. ${ }^{6}$

ACOG has noted 'Absence of visual image documentation eliminates the possibility of future review or clinical reintegration and weakens the defense against an allegation that an incomplete or inadequate study was performed' 3

\section{INDICATIONS}

The AIUM has published indications for first and second obstetric ultrasound examinations, which are listed in Tables 1 and $2 .^{5}$ When there is no indication, ACOG has commented that, while it is reasonable to honor a patient's request for an ultrasound, based upon the limitations of the various studies analyzing the benefits of routine screening and their equivocal results, a physician is not obligated to perform an ultrasound in a low-risk patient without indications. ${ }^{3}$ The authors have argued that all pregnant women should be offered a quality second trimester ultrasound examination in clinical settings where it is available. ${ }^{7}$ Further, it has been argued that pregnant women should also be offered a quality first trimester ultrasound examination in clinical settings where it is available. ${ }^{8}$ Currently, ACOG has recommended that all pregnant women, regardless of their age, should be offered screening for Down syndrome in a quality manner. ${ }^{9}$

\section{EXAMINATION CONTENT}

The AIUM has published a practice guideline for the performance of an antepartum obstetric ultrasound examination in conjunction with ACOG and the American College of Radiology (ACR). ${ }^{5}$ The components of a first

Table 1: First trimester ultrasound examination

Indications: A sonographic examination can be of benefit in many circumstances in the first trimester of pregnancy, including, but not limited to, the following indications:

- To confirm the presence of an intrauterine pregnancy

- To evaluate a suspected ectopic pregnancy

- To define the cause of vaginal bleeding

- To evaluate pelvic pain

- To estimate gestational (menstrual*) age

- To diagnose or evaluate multiple gestations

- To confirm cardiac activity

- As an adjunct to chorionic villus sampling, embryo transfer, and localization and removal of an intrauterine device (IUD)

- To evaluate maternal pelvic masses and/or uterine abnormalities

- To evaluate suspected hydatidiform mole.

*For the purpose of this document, the terms 'gestational age' and 'menstrual age' are considered equivalent.

AIUM Practice Guideline for the performance of an antepartum obstetric ultrasound examination. J Ultrasound Med 2003;22(10): 1116-25. Permission obtained. 
Table 2: Second- and third-trimester examination

Indications: Sonography can be of benefit in many situations in the second and third trimesters, including, but not limited to, the following circumstances: (adapted from National Institutes of Health. Diagnostic Ultrasound Imaging in Pregnancy: Report of a Consensus. NIH Publication 84-667. Washington, DC: US Government Printing Office; 1984).

- Estimation of gestational age

- Evaluation of fetal growth

- Vaginal bleeding

- Abdominal/pelvic pain

- Incompetent cervix

- Determination of fetal presentation

- Suspected multiple gestation

- Adjunct to amniocentesis

- Significant discrepancy between uterine size and clinical dates

- Pelvic mass

- Suspected hydatidiform mole

- Adjunct to cervical cerclage placement

- Suspected ectopic pregnancy

- Suspected fetal death

- Suspected uterine abnormality

- Evaluation of fetal well-being

- Suspected amniotic fluid abnormalities

- Suspected placental abruption

- Adjunct to external cephalic version

- Premature rupture of membranes and/or premature labor

- Abnormal biochemical markers

- Follow-up evaluation of a fetal anomaly

- Follow-up evaluation of placental location for suspected placenta previa

- History of previous congenital anomaly

- Evaluation of fetal condition in late registrants for prenatal care.

In certain clinical circumstances, a more detailed examination of fetal anatomy may be indicated.

AIUM Practice Guideline for the performance of an antepartum obstetric ultrasound examination. J Ultrasound Med. 2003;22(10): 1116-25. American Institute of Ultrasound in Medicine, publisher. Permission obtained.

trimester ultrasound examination and second and third trimester examinations are listed in Tables 3 and 4 . The AIUM and ACOG use the terms 'standard,' 'limited' and 'specialized' to describe the types of obstetric ultrasound performed during the second and third trimesters. Standard and limited examinations are defined by their components and the components of a specialized examination are determined on a case by case basis. ${ }^{3,5}$

Standard examinations include an evaluation of fetal presentation, amniotic fluid volume, cardiac activity, placental position, biometry and an anatomic survey. An examination of the uterus and adnexa is also suggested if technically feasible. ${ }^{3,5}$

Limited examinations are performed for a specific indication, such as identification of fetal presentation, evaluation of fetal cardiac activity or amount of amniotic fluid and are appropriate when a standard examination has already been performed. In such cases, an anatomic survey is not necessary.
Table 3: Contents of first trimester ultrasound examination

- Scanning in the first trimester may be performed either transabdominally or transvaginally. If a transabdominal examination is not definitive, a transvaginal scan or transperineal scan should be performed whenever possible.

- The uterus and adnexa should be evaluated for the presence of a gestational sac. If a gestational sac is seen, its location should be documented. The gestational sac should be evaluated for the presence or absence of a yolk sac or embryo and the crown-rump length should be recorded, when possible.

- Presence or absence of cardiac activity should be reported.

- Fetal number should be reported.

- Evaluation of the uterus, adnexal structures, and cul-de-sac should be performed.

Adapted from: AIUM Practice Guideline for the performance of an antepartum obstetric ultrasound examination. J Ultrasound Med 2003;22(10):1116-25. Permission obtained.

Table 4: Contents of a standard second and third trimester obstetric ultrasound examination

- Fetal cardiac activity, number and presentation should be reported.

- A qualitative or semiquantitative estimate of amniotic fluid volume should be reported.

- The placental location, appearance, and relationship to the internal cervical os should be recorded. The umbilical cord should be imaged and the number of vessels in the cord should be evaluated, when possible.

- Gestational age assessment.

- Fetal weight estimation.

- Maternal anatomy. Evaluation of the uterus and adnexal structures should be performed.

- Fetal anatomic survey.

- The following areas of assessment represent the essential elements of a standard examination of fetal anatomy. A more detailed fetal anatomic examination may be necessary if an abnormality or suspected abnormality is found on the standard examination.

- Head and neck

- Cerebellum

- Choroid plexus

- Cisterna magna

- Lateral cerebral ventricles

- Midline falx

- Cavum septi pellucidi

- Chest

- The basic cardiac examination includes a 4-chamber view of the fetal heart.

- If technically feasible, an extended basic cardiac examination can also be attempted to evaluate both outflow tracts.

- Abdomen

- $\quad$ Stomach (presence, size and sinus)

- Kidneys

- Bladder

- Umbilical cord insertion site into the fetal abdomen

- Umbilical cord vessel number

- Spine

- Cervical, thoracic, lumbar and sacral spine

- Extremities

- Legs and arms (presence or absence)

- Gender

- Medically indicated in low-risk pregnancies only for evaluation of multiple gestations.

Adapted from: AIUM Practice Guideline for the performance of an antepartum obstetric ultrasound examination. J Ultrasound Med 2003;22(10):1116-25. Permission obtained. 
Specialized examinations include the biophysical profile, fetal Doppler studies, fetal echocardiography and those examinations that are done when it is necessary to evaluate a specific question or to evaluate a specific or suspected fetal anomaly or maternal biochemical screening test. These examinations should be performed by operators with specific experience in the relevant area.,

\section{QUALITY CONTROL}

Following the results of the routine antenatal diagnostic imaging with ultrasound trial (RADIUS) published in $1993^{10}$ and other studies which indicated that the detection of anomalies was dependent on the experience of the operator, the AIUM began to offer voluntary medical facility accreditation for ultrasound practices. This process reviews the qualifications of the facility's practitioners, the type of equipment and its maintenance, including the proper methods of antimicrobial cleaning and/or chemical sterilization and storing of transducers to prevent contamination between patients, and methods of reporting and storage. ${ }^{11}$

The acquisition and maintainance of such accreditation ensures compliance with current organizational standards, is recommended and is often required for reimbursement for obstetric ultrasound studies by various insurance companies. A recent study in which practices that sought and received accreditation were re-evaluated 3 years later, found that these practices had improved compliance within accepted standards, and therefore concluded that this improvement would translate into an enhancement of the quality of practice. ${ }^{11}$

\section{LITIGATION RELATED TO ULTRASOUND}

Sanders had tracked litigation related to ultrasound. Since, there is no reliable system of tabulating legal cases that are filed, many cases are dropped following the review of a competent expert, the majority of cases settle out of court and not all of those that do go to court are reported, this task has been made especially difficult. In 2003, Sanders published his latest series documenting the types of cases he reviewed that were filed between 1997 and 2002. ${ }^{12}$

When categorizing suits by specialty, predictably those relating to obstetric ultrasound were the most common of all those involving ultrasound examinations, followed by gynecologic examinations. Suits relating to obstetric ultrasound can be expected to have large economic damages because damages are based upon the life-expectancy of the infant-plaintiff, thereby rewarding plaintiff's attorneys with large contingency fees. Sanders found that the missed fetal anomalies are now the most common reason for litigation,
Table 5: Nineteen possible ways to get sued for ultrasound

1. Missing the sonographic finding

2. Misinterpretation of the sonographic finding.

3. Failure to compare findings with previous ultrasound.

4. Failure to properly communicate the sonographic report to the referring physician or the patient.

5. Failure to personally examine the patient or take a proper history.

6. Incorrect sonographic approach for a specific condition.

7. Incomplete examination.

8. Inadequate quality of films.

9. Slip and fall injuries.

10. Complications from puncture techniques under ultrasound control.

11. Failure to obtain informed consent.

12. Complications of ultrasound such as induced vaginal bleeding or abortion.

13. Equipment complications (e.g. electric shocks).

14. Failure to recommend additional sonographic or radiologic studies or biopsy.

15. Failure to order a sonographic examination.

16. Inclusion of sinologist in a shotgun suit.

17. Loss of films, inadequate filing system, misplacement of films or reports.

18. Abuse of patient by sonologist or sonographer (sexual, physical or mental).

19. Miscellaneous anxiety produced by misdiagnosis, invasion of privacy, etc.

From: Sanders RC. The effect of the malpractice crisis on obstetrics and gynecologic ultrasound. Ultrasound in obstetrics and gynecology. In: Frank A. Chervenak, Glenn C Isaacson, et al (Eds). Boston: Little, Brown and Company 1993;263-67.

comprising over half of the cases in his most recent series. ${ }^{13}$ Table 5 documents Sander's tabulation of the possible ways to be sued when performing ultrasound. ${ }^{13}$

\section{NONMEDICAL USE OF ULTRASONOGRAPHY}

The AIUM has published the following 'prudent use' statement, endorsed by ACOG.

'The AIUM advocates the responsible use of diagnostic ultrasound. The AIUM strongly discourages the nonmedical use of ultrasound for psychosocial or entertainment purposes. The use of either two-dimensional or threedimensional ultrasound only to view the fetus, obtain a picture of the fetus or determine the fetal gender without a medical indication is inappropriate and contrary to responsible medical practice. Although there are no confirmed biological effects on patients caused by exposures from present diagnostic ultrasound instruments, the possibility exists that such biological effects may be identified in the future. Thus, ultrasound should be used in a prudent manner to provide medical benefit to the patient. ${ }^{14}$ This position has been ethically defended. ${ }^{15}$

\section{CONCLUSION}

Physicians who perform obstetric ultrasound can expect to be subjected to increasing legal risk. While knowledge of 
and comportment with the published recommendations and guidelines of ACOG and the AIUM does not offer complete protection from legal risk, they help to both avoid and to defend oneself. Failure to comply with such standards has the potential to make any subsequent legal case more difficult to defend.

\section{REFERENCES}

1. Prosser W, Keeton WP, Dobbs DB, et al. Prosser and Keeton on the Law of Torts (5th ed). Minnesota: West Publishing Group 1984;187.

2. Moore T, Gaier M. Medical malpractice. New York: Law Journal 2004:1.

3. Ultrasonography in pregnancy. ACOG Practice Bulletin(58), 2004. www.acog.org.

4. Guidelines for diagnostic imaging during pregnancy. ACOG Committee Opinion, 2004:299. www.acog.org.

5. AIUM practice guideline for the performance of an antepartum obstetric ultrasound examination. J Ultrasound Med 2003;22(10):1116-25.

6. American standard for documentation of an ultrasound examination. American Institute of Ultrasound in Medicine. J Ultrasound Med 2002:21(10):1188-89.

7. Chervenak FA, McCullough LB, Chervenak JL. Prenatal informed consent for sonogram (PICS): An indication for obstetric ultrasound. Am J Obstet Gynecol 1989;161:857-60.

8. Chasen S, Skupski DW, McCullough LB, Chervenak FA. Prenatal consent for sonogram: the time for first trimester nuchal translucency has come. J Ultrasound Med 2001;20:1147-52.

9. Laurie Barclay. Screening for fetal chromosomal abnormalities. Obstet Gynecol 2007.

10. Ewigman BG, Crane JP, Frigoletto FD, et al. Effect of prenatal ultrasound screening on perinatal outcome. RADIUS Study Group. N Engl J Med 1993;329(12):821-27.
11. Abuhamad AZ, Benacerraf B, Woletz P, et al. The accreditation of ultrasound practices-impact on compliance with minimum performance guidelines. J Ultrasound Med 2004;23(8):1023-29.

12. Sanders RC. Changing patterns of ultrasound-related litigation: A historical survey. J Ultrasound Med 2003;22(10):1009-15.

13. Sanders RC. The effect of the malpractice crisis on obstetrics and gynecologic ultrasound. In: Frank A Chervenak, Glenn C Isaacson, Stuart Campbell (Eds). Ultrasound in obstetrics and gynecology. Boston: Little Brown and Company 1993; 263-76.

14. Nonmedical use of obstetric ultrasonography. ACOG Committee Opinion, Nonmedical use of Obstetric Ultrasonography 2004;297.

15. Chervenak FA, McCullough LB. An Ethical critique of boutique fetal imaging: A case for the medicalization of fetal imaging. Am J Obstet Gynecol 2005;192(1):31-34.

\section{ABOUT THE AUTHORS}

\section{Frank A Chervenak}

Given Foundation Professor and Chairman, Obstetrician and Gynecologist-in-Chief, Department of Obstetrics and Gynecology Joan and Sanford I Weill Medical College of Cornell University, The New York Presbyterian Hospital, New York, USA.

\section{Judith L Chervenak}

Clinical Assistant Professor, Department of Obstetrics and Gynecology, New York University School of Medicine, New York, USA

\section{CORRESPONDING AUTHOR}

Frank A Chervenak, Department of Obstetrics and Gynecology, Weill Medical College of Cornell University, 525 East 68th Street, Box 122 New York, 10021, USA, Phone: 212-746-3045, Fax: 212-746-3012 e-mail: fac2001@med.cornell.edu 